\title{
Fish oil supplementation from 9 to 18 months of age affects the insulin-like growth factor axis in a sex-specific manner in Danish infants
}

\author{
Camilla T. Damsgaard ${ }^{1 *}$, Laurine B. S. Harsløf ${ }^{1}$, Anders D. Andersen ${ }^{2}$, Lars I. Hellgren ${ }^{3}$, \\ Kim F. Michaelsen ${ }^{1}$ and Lotte Lauritzen ${ }^{1}$ \\ ${ }^{1}$ Department of Nutrition, Exercise \& Sports, Faculty of Science, University of Copenhagen, DK-1958 Frederiksberg C, Denmark \\ ${ }^{2}$ Department of Veterinary Clinical and Animal Sciences, Faculty of Health and Medical Sciences, University of Copenhagen, \\ DK-1958 Frederiksberg C, Denmark \\ ${ }^{3}$ Department of Systems Biology, Center for Biological Sequence Analysis, Technical University of Denmark, 2800 Lyngby, Denmark \\ (Submitted 8 May 2015 - Final revision received 5 October 2015 - Accepted 13 November 2015 - First published online 13 January 2016)
}

\begin{abstract}
Several studies have investigated the effects of fish oil (FO) on infant growth, but little is known about the effects of FO and sex on insulin-like growth factor-1 (IGF-1), the main regulator of growth in childhood. We explored whether FO $v$. sunflower oil (SO) supplementation from 9 to 18 months of age affected IGF-1 and its binding protein-3 (IGFBP-3) and whether the potential effects were sex specific. Danish infants ( $n$ 115) were randomly allocated to $5 \mathrm{ml} / \mathrm{d}$ FO $(1.2 \mathrm{~g} / \mathrm{d} n-3$ long-chain PUFA ( $n-3$ LCPUFA)) or SO. We measured growth, IGF-1, IGFBP-3 and erythrocyte EPA, a biomarker of $n$-3 LCPUFA intake and status, at 9 and 18 months. Erythrocyte EPA increased strongly with FO compared with SO $(P<0 \cdot 001)$. There were no effects of FO compared with SO on IGF-1 in the total population, but a sex $\times$ group interaction $(P=0 \cdot 02)$. Baseline-adjusted IGF-1 at 18 months was $11 \cdot 1 \mu \mathrm{g} / \mathrm{l}(95 \% \mathrm{CI} 0 \cdot 4,21 \cdot 8 ; P=0 \cdot 04)$ higher after FO compared with SO supplementation among boys only. The sex $\times$ group interaction was borderline significant in the model of IGFBP-3 $(P=0 \cdot 09)$, with lower IGFBP-3 with FO compared with SO among girls only $(P=0 \cdot 03)$. The results were supported by sex-specific dose-response associations between changes in erythrocyte EPA and changes in IGF-1 and IGFBP-3 (both $P<0 \cdot 03$ ). Moreover, IGF-1 was sex specifically associated with BMI and length. In conclusion, FO compared with SO resulted in higher IGF-1 among boys and lower IGFBP-3 among girls. The potential long-term implications for growth and body composition should be investigated further.
\end{abstract}

Key words: Growth: PUFA: Children: Obesity: Sex differences

Insulin-like growth factor-1 (IGF-1) is the main predictor of height velocity in childhood $^{(1)}$. During early infancy, IGF-I formation is mainly regulated by nutrition, but a gradual transition to regulation of IGF-I by growth hormone occurs around the ages of 6-12 months ${ }^{(2)}$. More than $90 \%$ of the IGF-1 in plasma is bound to IGF-1 and its binding protein-3 $\left(\right.$ IGFBP-3) ${ }^{(3)}$, and the IGF-1:IGFBP-3 ratio is thought to reflect the concentration of free, biologically active IGF- ${ }^{(4)}$. IGF-1 mediates the effects of growth hormone on bone mineralisation, linear growth and muscle mass accretion in childhood by endocrine, paracrine and autocrine signalling ${ }^{(4)}$. IGF-1 signals through binding to the IGF-1 receptor, and it can also bind to insulin receptors, although with lower affinity ${ }^{(4)}$, thereby sharing some of insulin's anabolic effects. Plasma IGF-1 has been shown to be higher in girls than in boys ${ }^{(5)}$ and to be modulated by protein and fat in the diet ${ }^{(6)}$. IGF-1 has also been associated with obesity in early life, but the relation is complex and differs with age ${ }^{(6)}$.
Animal studies and randomised trials mainly in postmenopausal women indicate that $n-3$ long-chain PUFA ( $n$-3 LCPUFA) from fatty fish and fish oils (FO) can increase markers of bone formation and reduce bone loss ${ }^{(7-9)}$. We recently showed that FO compared with sunflower oil (SO) feeding increased bone mineral content evaluated by dual-energy X-ray absorptiometry (DXA) in growing piglets ${ }^{(10)}$. In addition, there is some evidence that $n$ - 3 LCPUFA supplementation can modulate IGF-1 ${ }^{(11,12)}$, as well as IGF-binding proteins and receptors, in young animals ${ }^{(11)}$. We recently found no effects of FO supplementation on bone mass or on serum IGF-1, IGFBP-3 or Osteocalcin in Danish teenage boys ${ }^{(13)}$. However, in that study, changes in plasma IGF-1 during the intervention were positively associated with changes in the content of $n$-3 LCPUFA in erythrocytes, a biomarker of the $n$ - 3 LCPUFA intake during the supplementation.

Most $n$-3 LCPUFA studies have been conducted in males or without specific attention to sex, but recent work indicate that

Abbreviations: FO, fish oil; IGF-1, insulin-like growth factor-1; IGFBP-3, IGF-1 and its binding protein-3; n-3 LCPUFA, $n-3$ long-chain PUFA; SO, sunflower oil. 
some effects of $n$-3 LCPUFA in children may be sex specific $^{(14,15)}$. Maternal $n-3$ LCPUFA supplementation in rats has been shown to affect bone marrow microenvironment and osteoblast and osteoclast formation among male offspring only $^{(16)}$. The effect was seen at 3 weeks of age but not at later ages. In a Danish cohort of breast-fed infants, DHA (22:6n-3) in maternal breast milk was inversely associated with BMI accretion from 2 to 7 years of age and associated with higher age at adiposity rebound in girls, but not in boys ${ }^{(17)}$. However, the potential sex-specific effects of $n-3$ LCPUFA on IGF- 1 and its binding proteins have, to our knowledge, not been investigated in children or adults. We previously found no effects of FO supplementation from 9 to 18 months of age on growth in Danish children; however, at that time we did not look at potential sex-specific effects or effects on the IGF axis ${ }^{(18)}$.

The aim of this paper was to explore whether supplementation with FO $v$. SO from 9 to 18 months of age affected IGF-1 and IGFBP-3 in Danish infants and to assess whether potential effects were different in boys and girls.

\section{Methods \\ Study design and subjects}

The EFiON (Essentielle Fedtsyrer i OvergangskosteN) study was a randomised, controlled trial in which infants were allocated to a daily oil supplement from 9 to 18 months of age. The study design and methods were previously described in detail by Andersen et $a l .{ }^{(18)}$, and other results from this study have been published previously ${ }^{(18-22)}$. The study was designed to investigate the effects of $n$-3 LCPUFA on growth and body composition during the complementary feeding period $^{(18)}$. The study was conducted at Department of Nutrition, Exercise and Sports, University of Copenhagen from January 2008 to March 2009, it was approved by the Scientific Ethics Committees of the Capital Region of Denmark (no. H-A-2007-0088) and registered at ClinicalTrials.gov as NCT 00631046. All procedures involving human subjects were conducted according to the guidelines in the Declaration of Helsinki. A total of 154 infants were included in the EFiON study, and 133 infants completed the trial ${ }^{(18)}$. The 115 infants (fifty-nine boys and fifty-six girls) who were successfully blood sampled at both 9 and 18 months were included in this study.

In short, families with 8-month-old infants in the Capital Region of Denmark were invited for participation through the National Danish Civil Registry and received written information about the study. Parents who wished to participate were invited to an individual information meeting and gave written informed consent for participation. Eligible infants were healthy singletons, born $>37$ th week of gestation and with appropriate weight for gestational age ${ }^{(18)}$. Infants were excluded if they had previously received FO supplements, had chronic diseases or had received medication that could influence growth and/or food intake.

\section{Intervention}

The infants were randomised to receive $5 \mathrm{ml} / \mathrm{d}$ FO (Möller's $n$ - 3 cod liver oil without vitamins) or $5 \mathrm{ml} / \mathrm{d}$ SO (Bressmer's sunflower oil), both produced and kindly provided by Axellus A/S, Lysaker, Norway. Group allocation was performed by blinded personnel based on a computer-generated block randomisation with varying block sizes of 4, 6, 8 and 10 (www. randomization.com). The parents were asked to keep the unopened oil bottles in the freezer and to keep the bottles refrigerated once opened to minimise fatty acid oxidation and odours, as the oils were odourless at these temperatures. They were asked to give the oil on a spoon or add it to the infant's porridge/mash instead of the fat normally added before serving it; however, no specific instructions on avoidance of heating were given. The oils were provided in ten dark 150-ml bottles of similar appearance ( 1.5 litre in total), and the empty bottles including oil leftovers were returned for assessment of compliance at the 18-month examination. To check the blinding of parents, the contact parent was asked to guess the child's allocated oil type by the end of the study. The supplements were estimated to provide about $3.5 \%$ of the infants' mean energy requirement. The SO intake supplied $2.4 \mathrm{~g} / \mathrm{d}$ linoleic acid (LA) (18:2n-6), and the added LA accounted for about $2.4 \%$ of the energy intake (E\%). The FO supplied $1.2 \mathrm{~g} / \mathrm{d}$ or about $1.4 \mathrm{E} \% n$-3 LCPUFA, with approximately equal contribution of EPA $(20: 5 n-3)$ and $\mathrm{DHA}^{(18)}$.

\section{Measurements}

Detailed questionnaires on socio-demographics and other characteristics were completed before the examinations at 9 and 18 months. Information on the daily food intake of the infants was obtained by validated pre-coded food diaries filled in by the parents during 7 consecutive days before the two examinations $^{(23)}$.

Anthropometric measurements were performed by trained investigators, as described previously ${ }^{(18)}$. Recumbent length was measured to the nearest millimetre on a wooden measuring board (FORCE Technology). A total of three measurements were conducted, and the mean value of the measurements was used. Weight was measured on a paediatric infant scale (Sartorius IP65; Bie \& Berntsen AS). If the infant weighed $\geq 12 \mathrm{~kg}$, the infant was weighed with a parent on an adult scale (Lindeltronic 8000; Samhall Lavi AB) with subsequent subtraction of parent weight. Triceps and subscapular skinfold thicknesses were measured to the nearest $0 \cdot 1 \mathrm{~mm}$ (Harpenden skinfold caliper; CMS Weighing Equipment Ltd). The interobserver variability for triceps skinfolds measurements was $<0.5 \mathrm{~mm}^{(18)}$.

\section{Blood sampling and analysis}

Venous blood was sampled from the infant's forearm. Parents were instructed to apply local anaesthetic patches (EMLA; AstraZeneca $\mathrm{AB}$ ) before blood sampling and were instructed to keep their child from eating at least $2 \mathrm{~h}$ before blood sampling. Parent-reported mean fasting time was approximately 157 (sD 47) and 150 (SD 33) min at 9 and 18 months, with the exception of three infants who had fasted $14-16 \mathrm{~h}$ before the 18-month examination. 
Erythrocytes were isolated from heparinised blood samples, washed with saline and kept at $-80^{\circ} \mathrm{C}$ for later GC analysis, as previously described by Lauritzen et $a l^{(24)}$. The contents of specific fatty acids were calculated as area\% of the specific fatty acid relative to the total identified chromatogram area (FA\%). Plasma IGF-1 and IGFBP-3 were determined in heparinised plasma by automated assays on an Immulite 1000 (Siemens Healthcare Diagnostics). Plasma IGF-1 concentrations below the detection limit $(<25 \mathrm{ng} / \mathrm{ml})$ were defined as $12.5 \mathrm{ng} / \mathrm{ml}$ ( $n$ 10). To estimate the IGF-1:IGFBP-3 molar ratio, the following conversion equivalents were used: $1 \mu \mathrm{g} / 1$ IGF- $1=0.133 \mathrm{~nm}$ IGF-1 and $1 \mathrm{mg} / 1$ IGFBP-3 = 33 nм IGFBP-3.

\section{Statistical analysis}

Data are presented as mean values and standard deviations or medians and interquartile ranges as appropriate. Only participants with available outcome data from both examinations were included in the analyses (complete case analysis). Outcome differences between intervention groups were evaluated in ANCOVA. Stepwise backward model reduction was used to remove interaction terms and covariates from a fully adjusted model to a final model, for each outcome. Covariates that were likely to affect the outcome $(P<0 \cdot 10)$ were kept in the model. For all outcomes, the full model initially included the following: baseline outcome values, oil compliance $(\mathrm{ml} / \mathrm{d})$, breast-feeding during the intervention (yes/no) and a sex (girl/boy) $\times$ group $(\mathrm{FO} / \mathrm{SO})$ interaction term. Baseline outcome values and oil compliance were kept as covariates in all final models. Simple group comparisons were performed using unpaired $t$ test and Mann-Whitney $U$ test for normally and non-normally distributed variables, respectively. Bivariate correlations and dose-response analyses were performed using Pearson's Product Moment correlation. Data were analysed using SPSS (version 22; IBM Corporation), and significance was established at $P<0.05$; however, the sex $\times$ group interaction was explored further at $P<0 \cdot 10$ (borderline significant). Because the EFiON study was the first of its kind, we were unable to make proper estimates of the predicted effect size on the anthropometric end points. However, with 130 infants completing the trial, we would be able to detect group differences of about $0.5 \mathrm{sD}$, with a power of 0.80 and $\alpha=0.05^{(18)}$.

\section{Results}

\section{Baseline characteristics}

Of the 154 children originally included in the EFION study, 115 children had blood analyses available from both 9 and 18 months and were included in the present study. Included and non-included children did not differ with regard to age, length or BMI at baseline $(P>0 \cdot 17$, unpaired $t$ tests). Table 1 shows the characteristics of the included infants at birth and at baseline, where about half of the children were still partially breast-fed and most children had started eating small amounts of fish. The full erythrocyte fatty acid composition of the infants was published previously ${ }^{(18)}$
Girls had higher IGF-1:IGFBP-3 $(P=0.02)$ than boys at baseline and also tended to have $15 \%$ higher IGF-1 (54.9 (SD 18.7) v. 47.9 (SD 20.4) $\mu \mathrm{g} / \mathrm{l} ; P=0.06$ ). There were no sex differences in the major PUFA, EPA or DHA in erythrocytes at baseline $(P>0.23)$. In addition, neither erythrocyte EPA nor DHA were cross-sectionally associated with IGF-1, IGFBP-3 or their molar ratio at baseline in the total population or in the sexes separately (data not shown). Fasting time before blood sampling was not associated with any of the IGF axis measures, either at baseline or at 18 months (data not shown).

\section{Compliance and parent blinding}

The median intakes of $\mathrm{FO}$ and SO were 3.7 (IQR 3.0-4.2) and $3 \cdot 8$ (IQR $3 \cdot 3-4 \cdot 2$ ) ml/d, corresponding to 74 and $76 \%$ compliance in the FO and SO groups, respectively, and did not differ between the groups ( $P=0 \cdot 31$, Mann-Whitney $U$ test). In addition, oil intake did not differ between boys and girls either in the total population or within each intervention group ( $P>0 \cdot 17$, Mann-Whitney $U$ tests). As previously shown, the erythrocyte content of EPA and DHA increased in the FO group during the intervention by 5.5 (IQR 4.1-7.4) and $4 \cdot 8$ (sD 0.3) FA\%, respectively, both $P<0.001$ compared with $\mathrm{SO}^{(18)}$. Additional analyses showed that the change in erythrocyte EPA during the intervention was 0.78 (95\% CI $0.02,1.53$ ) FA\% larger in girls compared with boys in the overall population $(P=0.04$ by ANOVA), but this difference did not depend on the intervention group $\left(P_{\text {sex } \times \text { group }}=0 \cdot 70\right)$. No sex differences or $\operatorname{sex} \times$ group interactions were seen for changes in erythrocyte DHA, LA or arachidonic acid during the intervention $(P>0 \cdot 21)$.

When asked by the end of the intervention, 84 and $88 \%$ of the parents in the FO and SO group, respectively, were able to successfully guess their child's allocated oil type, indicating that parent blinding was partly unsuccessful.

\section{Effects of the intervention}

There were no differences in IGF-1, IGFBP-3 or their molar ratio between the FO and SO groups in the total study population (Table 2). However, in the model of IGF-1, there was a significant sex $\times$ group interaction $(P=0.02)$, and the interaction term was borderline significant in the models of IGFBP-3 $(P=0.09)$ and IGF-1:IGFBP-3 $(P=0.08)$ (Table 2$)$. Analysing data for boys and girls separately showed that FO compared with SO gave $11.1 \mu \mathrm{g} / \mathrm{l}$ (95\% CI $0.4,21 \cdot 8 ; P=0.04)$ higher baseline-adjusted IGF-1 concentrations at 18 months among the boys only, whereas IGFBP-3 was $0.27 \mathrm{mg} / \mathrm{l}$ (95\% CI 0.03, 0.51; $P=0.03$ ) lower among the girls (Table 2). Accordingly, IGF-1: IGFBP-3 tended to be higher with FO compared with SO among boys $(P=0 \cdot 10)$, but not among girls $(P=0 \cdot 63)$ (Table 2$)$. Children who were still breast-fed at 9 months tended to have lower IGF-1 at this age compared with children who were no longer breast-fed (sex-adjusted mean difference $-7.2 \mu \mathrm{g} / 1$ (95\% CI $-14.4,0 \cdot 1 ; P=0 \cdot 06$ ). However, breast-feeding during the intervention did not have any effects on the IGF axis measures and was therefore not included in the final models. In addition, there were no differences between the groups or sex $\times$ group interactions in intakes of protein, fat or 
Table 1. Baseline characteristics of the study population according to intervention group (Mean values and standard deviations; medians and interquartile ranges (IQR))

\begin{tabular}{|c|c|c|c|c|}
\hline & \multicolumn{2}{|c|}{$\mathrm{FO}(n 56)$} & \multicolumn{2}{|c|}{ SO (n 57-59) } \\
\hline & Mean & SD & Mean & SD \\
\hline \multicolumn{5}{|l|}{ Birth characteristics and breast-feeding } \\
\hline Sex $(M: F)$ & & & & \\
\hline Gestational length (weeks) & 40.0 & 1.5 & $40 \cdot 0$ & 1.4 \\
\hline Birth weight $(\mathrm{kg})$ & 3.5 & 0.4 & 3.6 & 0.6 \\
\hline Birth length $(\mathrm{cm})$ & 52.0 & 1.9 & $52 \cdot 4$ & $2 \cdot 4$ \\
\hline \multicolumn{5}{|l|}{ Duration of full breast-feeding (months) } \\
\hline Median & & & & \\
\hline IQR & & & & \\
\hline Still breast-fed at 9 months (\% yes) & & & & \\
\hline \multicolumn{5}{|l|}{ Anthropometry at 9 months } \\
\hline Age (months) & 9.1 & 0.3 & $9 \cdot 1$ & 0.3 \\
\hline Weight $(\mathrm{kg})$ & $9 \cdot 3$ & 1.0 & $9 \cdot 1$ & 1.0 \\
\hline Length $(\mathrm{cm})$ & $72 \cdot 2$ & 2.4 & $72 \cdot 1$ & $2 \cdot 3$ \\
\hline BMI $\left(\mathrm{kg} / \mathrm{m}^{2}\right)$ & 17.9 & 1.5 & 17.5 & 1.4 \\
\hline \multicolumn{5}{|c|}{ Dietary intake at 9 months (not including breast milk) } \\
\hline Energy intake $(\mathrm{kJ} / \mathrm{d})$ & 3195 & 1134 & 3087 & 800 \\
\hline Protein intake (E\%) & $12 \cdot 4$ & 1.9 & $12 \cdot 4$ & $1 \cdot 8$ \\
\hline Carbohydrate intake ( $\mathrm{E} \%)$ & $50 \cdot 4$ & 4.7 & $51 \cdot 1$ & 5.9 \\
\hline Fat intake (E\%) & 37.2 & 4.6 & 36.5 & $5 \cdot 6$ \\
\hline SFA (E\%) & $12 \cdot 8$ & 3.9 & $14 \cdot 2$ & $4 \cdot 1$ \\
\hline MUFA (E\%) & $12 \cdot 7$ & 3.0 & $12 \cdot 1$ & 2.6 \\
\hline \multicolumn{5}{|l|}{ PUFA (E\%) } \\
\hline Median & & & & \\
\hline IQR & & & & \\
\hline \multicolumn{5}{|l|}{$n-3$ PUFA (E\%) } \\
\hline Median & & & & \\
\hline IQR & & & & \\
\hline \multicolumn{5}{|l|}{$n-6$ PUFA (E\%) } \\
\hline Median & & & & \\
\hline IQR & & & & \\
\hline \multicolumn{5}{|l|}{$n-6: n-3$ PUFA } \\
\hline Median & & & & \\
\hline IQR & & & & \\
\hline Fish introduced at 9 months (\% yes) & & & & \\
\hline \multicolumn{5}{|l|}{ Fish intake $(\mathrm{g} / \mathrm{d})$} \\
\hline Median & & & & \\
\hline IQR & & & & \\
\hline \multicolumn{5}{|c|}{ Erythrocyte fatty acid composition at 9 months } \\
\hline \multicolumn{5}{|c|}{ EPA $20: 5 n-3(F A \%)$} \\
\hline Median & & & & \\
\hline IQR & & & & \\
\hline DHA $22: 6 n-3$ (FA\%) & 6.64 & 1.83 & 6.62 & 1.56 \\
\hline
\end{tabular}

FO, fish oil; SO, sunflower oil; M, male; F, female; E\%, percentage of the energy intake; FA\%, percentage of the fatty acids.

carbohydrate at 18 months adjusted for baseline values (data not shown).

No effects of the intervention or sex $\times$ group interactions were found for length or BMI, although there was a slight tendency for higher length after the intervention in the FO group compared with the SO group (estimated difference $0.4 \mathrm{~cm}(95 \% \mathrm{CI}-0 \cdot 8$, $0 \cdot 1 ; P=0 \cdot 11$ ), adjusted for sex and baseline length) (Table 2).

\section{Dose-response associations}

Changes from 9 to 18 months in erythrocyte EPA showed stronger correlation with the estimated FO consumption, compared with changes in erythrocyte DHA $(r 0.59, P<0.01$ for EPA and $r 0.09, P=0.52, n 55$ for DHA). Accordingly, the change in erythrocyte EPA was used as an objective indicator of oil consumption in the dose-response analyses.
Changes in IGF-1 from 9 to 18 months were positively correlated with changes in erythrocyte EPA in boys, but not in girls (Fig. 1). Moreover, changes in IGFBP-3 were negatively correlated with changes in erythrocyte EPA in girls, not in boys (Fig. 2). Using changes in erythrocyte DHA rather than EPA in the correlations showed the same overall pattern (data not shown).

\section{Associations between the insulin-like growth factor axis and growth}

Among the boys, IGF-1 (Fig. 3) and IGF-1:IGFBP-3 ( $r$ 0.49, $P<0.001)$ at 18 months were positively correlated with length at 18 months, but such correlations were not seen among girls (Fig. 3; and $r 0 \cdot 10, P=0.45$ for IGF-1:IGFBP-3). In addition, IGF-1 and IGF-1:IGFBP-3 were positively correlated with BMI at 18 months in boys $(r 0.33, P=0.01$ and $r 0.32, P=0.02$, 
Table 2. Plasma insulin-like growth factor-1 (IGF-1), and its binding protein-3 (IGFBP-3) and selected anthropometric measures before and after the intervention in the fish oil (FO) and sunflower oil (SO) groups shown separately in boys and girls

(Mean values and standard deviations)

\begin{tabular}{|c|c|c|c|c|c|c|c|c|c|c|c|c|}
\hline & \multicolumn{5}{|c|}{ Boys } & \multicolumn{5}{|c|}{ Girls } & & \\
\hline & \multicolumn{2}{|c|}{$\mathrm{FO}(n 26)$} & \multicolumn{2}{|c|}{ SO (n 33) } & \multirow[b]{2}{*}{$P_{\text {group }}{ }^{*}$} & \multicolumn{2}{|c|}{$\mathrm{FO}(n 30)$} & \multicolumn{2}{|c|}{$\mathrm{SO}(n 26)$} & \multirow[b]{2}{*}{$P_{\text {group }}{ }^{*}$} & \multicolumn{2}{|c|}{ Total population } \\
\hline & Mean & SD & Mean & SD & & Mean & SD & Mean & SD & & $P_{\text {group } \times \text { sex }^{*}}$ & $P_{\text {group }}{ }^{*}$ \\
\hline \multicolumn{13}{|c|}{ Plasma IGF-1 $(\mu \mathrm{g} / \mathrm{I}) \dagger$} \\
\hline 9 months & $45 \cdot 8$ & $22 \cdot 0$ & 49.5 & $19 \cdot 3$ & & 47.9 & $20 \cdot 3$ & $54 \cdot 3$ & $16 \cdot 9$ & & & \\
\hline 18 months & $60 \cdot 5$ & $32 \cdot 8$ & $52 \cdot 7$ & $22 \cdot 5$ & 0.04 & $66 \cdot 4$ & $20 \cdot 7$ & $74 \cdot 3$ & $25 \cdot 5$ & 0.17 & 0.02 & - \\
\hline \multicolumn{13}{|c|}{ Plasma IGFBP-3 (mg/l) } \\
\hline 9 months & $2 \cdot 51$ & 0.69 & 2.50 & 0.59 & & $2 \cdot 61$ & 0.62 & 2.57 & 0.54 & & & \\
\hline 18 months & $2 \cdot 52$ & 0.67 & 2.47 & 0.66 & 0.48 & $2 \cdot 61$ & 0.49 & $2 \cdot 85$ & 0.56 & 0.03 & 0.09 & 0.21 \\
\hline \multicolumn{13}{|c|}{ Plasma IGF-1:IGFBP-3 } \\
\hline 9 months & 0.07 & 0.02 & 0.08 & 0.03 & & 0.09 & 0.02 & 0.09 & 0.02 & & & \\
\hline 18 months & 0.09 & 0.03 & 0.09 & 0.03 & 0.10 & $0 \cdot 10$ & 0.03 & 0.11 & 0.03 & 0.63 & 0.08 & 0.34 \\
\hline \multicolumn{13}{|l|}{ Length (cm) } \\
\hline 9 months & 73.4 & $2 \cdot 4$ & $72 \cdot 8$ & $2 \cdot 1$ & & 71.1 & 1.8 & 71.3 & $2 \cdot 3$ & & & \\
\hline 18 months & $83 \cdot 2$ & $3 \cdot 2$ & $82 \cdot 3$ & $2 \cdot 7$ & - & 81.5 & $2 \cdot 3$ & $81 \cdot 3$ & $2 \cdot 3$ & - & 0.99 & $0 \cdot 11$ \\
\hline \multicolumn{13}{|l|}{ BMI $\left(\mathrm{kg} / \mathrm{m}^{2}\right)$} \\
\hline 9 months & $17 \cdot 9$ & 1.2 & $17 \cdot 8$ & 1.5 & & $17 \cdot 9$ & 1.7 & $17 \cdot 1$ & $1 \cdot 1$ & & & \\
\hline 18 months & $17 \cdot 1$ & 1.4 & $17 \cdot 3$ & 1.4 & - & $17 \cdot 0$ & 1.4 & $16 \cdot 5$ & $1 \cdot 1$ & - & 0.69 & 0.33 \\
\hline
\end{tabular}

* ANCOVA of 18-month values adjusted for 9-month values and intake of intervention oils. When sex $\times$ group was borderline significant in the model $(P<0 \cdot 10)$, group effects were tested both in the total population and separately in boys and girls.

† Plasma IGF-1 concentrations below the detection limit $(<25 \mu \mathrm{g} / \mathrm{l})$ were defined as $12.5 \mu \mathrm{g} / \mathrm{l}(n 10)$.
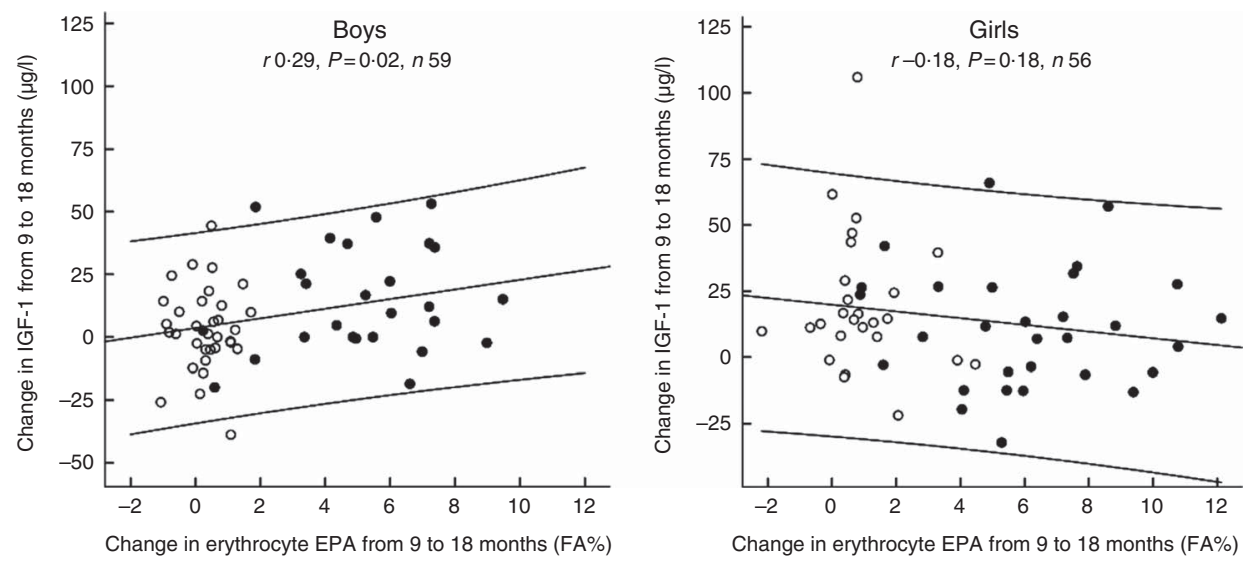

Fig. 1. Correlations between changes in erythrocyte EPA and changes in plasma insulin-like growth factor-1 (IGF-1) from 9 to 18 months in boys and girls. $O$, Sunflower oil; $\bullet$, fish oil. FA\%, percentage of fatty acids.
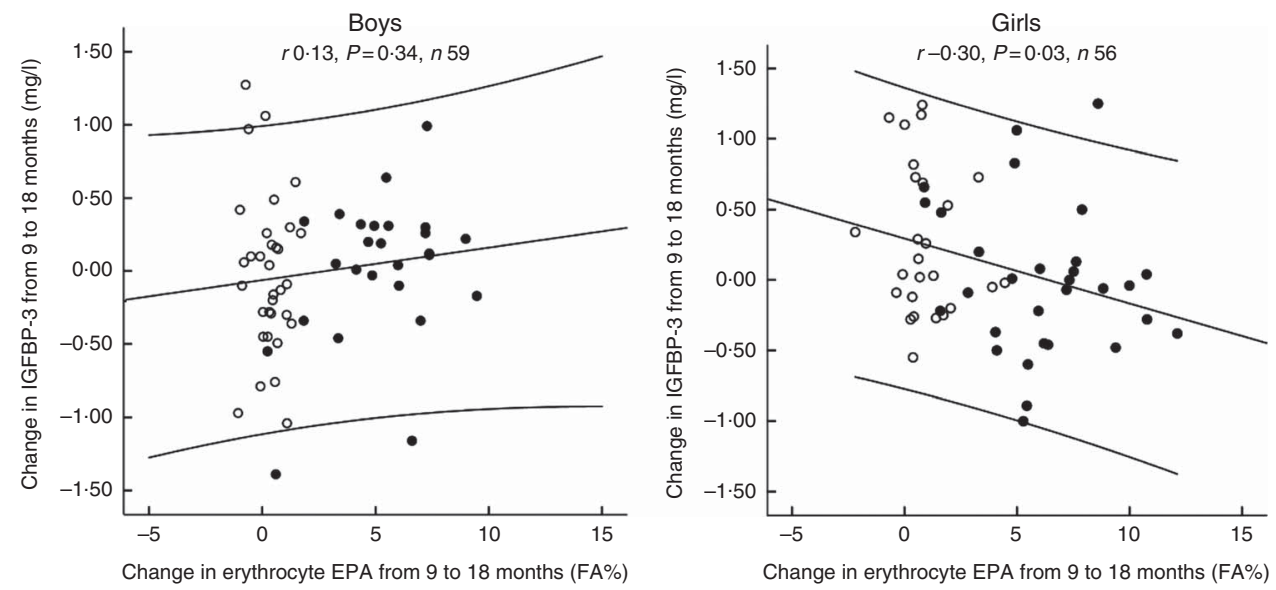

Fig. 2. Correlations between changes in erythrocyte EPA and changes in plasma insulin-like growth factor binding protein-3 (IGFBP-3) from 9 to 18 months in boys and girls. $\mathrm{O}$, Sunflower oil; $\boldsymbol{\bullet}$, fish oil. FA\%, percentage of fatty acids. 

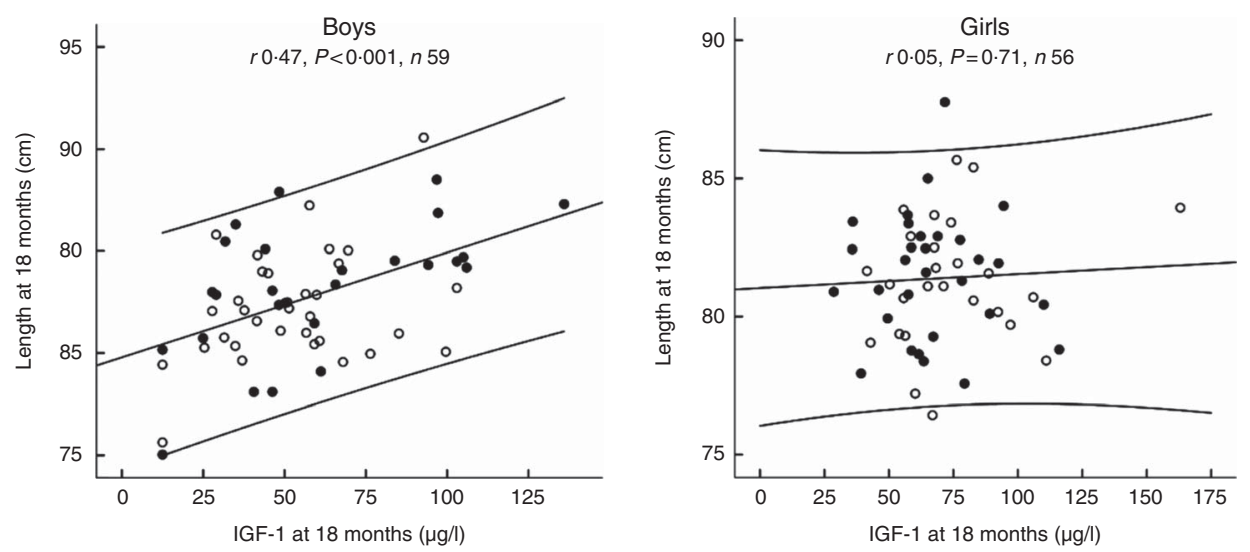

Fig. 3. Correlations between plasma insulin-like growth factor-1 (IGF-1) and infant length at 18 months in boys and girls. $\bigcirc$, Sunflower oil; $\bullet$, fish oil.

respectively), but not in girls $(r 0.21, P=0.13$ and $r 0.15$, $P=0 \cdot 27$, respectively). Changes during the intervention in IGF-1 were not correlated with changes in length in either boys or girls, but were positively correlated with changes in BMI in boys $(r 0.27, P=0.04)$ and even more pronounced in girls ( $r 0.46, P<0 \cdot 001$ ) (online Supplementary Fig. S1).

\section{Discussion}

This randomised controlled trial showed that although there were no effects of the intervention on the IGF axis in the total study population, FO $v$. SO supplementation from 9 to 18 months of age affected the IGF axis in a sex-specific manner in Danish infants. Plasma IGF-1 was higher in boys and IGFBP-3 was lower in girls after FO compared with SO supplementation. The results were supported by dose-response analyses, and although no effects were seen on growth outcomes IGF-1 was positively associated with both length and BMI at 18 months in the boys. To our knowledge, no previous trials have investigated sex-specific effects of $n$-3 LCPUFA on the IGF axis.

Our findings are in line with the observed association between IGF-1 and erythrocyte DHA after FO supplementation in our previous study in teenage boys ${ }^{(13)}$. However, in our previous infant trial, there was no effect of FO supplementation from 9 to 12 months of age on IGF-1, and also no interaction effect between sex and FO on IGF-1 or the anthropometric outcomes $^{(25)}$. However, that study had a shorter intervention period than the current study, and no control oil was used. A randomised controlled trial among men with CVD showed that a relatively high dose of FO ( $1.2 \mathrm{~g} / \mathrm{d} n$-3 LCPUFA) for 8 weeks increased serum IGF-1 and reduced IGFBP-3, with a resulting reduction in their molar ratio ${ }^{(26)}$. In growing pigs, supplementation with DHA compared with soyabean oil (an n-6 PUFA-rich oil) increased $I G F-1$ expression in skeletal muscle, probably indicative of stimulated muscle protein synthesis, but it did not affect plasma IGF-1 concentrations or hepatic $I G F-1$ expression ${ }^{(12)}$. In addition, we have recently shown that FO compared with SO feeding increased bone mineral content in growing piglets ${ }^{(10)}$. However, although $n-3$ LCPUFA supplementation to pre-term infants has been shown to increase length at 18 months of age ${ }^{(27)}$, a meta-analysis by Makrides et $a l^{(28)}$ concluded that supplementation of infant formula with $n$-3 LCPUFA did not affect growth of term infants at 4 or 12 months of age. Moreover, the meta-analysis did not show sex-specific effects of FO on growth. It should be noted, however, that the included trials administered $n$ - 3 LCPUFA in lower doses $(0 \cdot 1-1 \cdot 0 \%$ of total fatty acids compared with $1.2 \mathrm{~g} / \mathrm{d}$ or about $10 \%$ of total fatty acids in the present study) and at an earlier age than in the present study.

In the present study, children were supplemented with FO or SO from 9 to 18 months of age, a period during which plasma IGF-1 and IGFBP-3 concentrations are expected to increase steadily $^{(6)}$. Circulating IGF-1 is known to stimulate bone growth and muscle mass accretion, and it shows higher concentrations in girls than in boys in most cohorts ${ }^{(5,29)}$, despite the slower linear growth of girls. In line with this, baseline IGF-1 tended to be higher in girls than in boys in the present study, and this was probably a biological sex characteristic, rather than being related to fatty acid status, as no sex differences in PUFA or $n-3$ LCPUFA status were present at baseline. IGF-1 and IGFBP-3 at 18 months were positively associated with length at 18 months in boys only, indicating that IGF-1 may affect growth differently in boys and girls.

As also described for the total study population in our previous publication $^{(18)}$, FO did not affect linear growth or BMI of children, which we would expect with the effects on the IGF axis, at least in boys. The long-term implications of the results are therefore unknown; however, we cannot exclude the possibility that the higher IGF-1 concentrations with FO supplementation may accelerate linear growth or body mass accretion later in childhood. Although we did observe a larger increase in erythrocyte EPA during the intervention in girls compared with boys, this is unlikely to explain the sex-specific changes in IGF-1 during the intervention, as the sex difference in the EPA increase did not differ between the FO and SO groups, and because there were no sex differences in the amounts of intervention oils consumed. It is possible that the lack of IGF-1 increase with FO in the girls could be related to the already high plasma IGF-1 in the girls at baseline, which may not have been able to increase further. Anyway, we note that the different effects of FO on the IGF axis in boys and girls 
in the present study seemed to counteract the sex differences present at baseline. This is in line with the apparent 'sex-equalising' effects of FO that we have seen previously in relation to blood pressure ${ }^{(14)}$ and others have seen in relation to cognitive outcomes ${ }^{(30)}$.

There is some evidence that higher IGF-1 concentrations and faster growth during early infancy are associated with a higher risk of obesity and cardiometabolic disturbances in later life ${ }^{(6)}$. Although no effects of the intervention were seen on BMI, IGF-1 at 18 months was positively associated with BMI in boys and with changes in BMI in both sexes. Unfortunately, we were not able to assess potential effects on lean body mass. The tendency of lower baseline IGF-1 in children who were still breast-fed compared with those who were no longer breast-fed at 9 months is probably related to the lower protein content of breast milk compared with formula and cows' milk, rather than to the high content of DHA in breast milk. In line with this, the group of Koletzko has demonstrated that infants randomised to formula with high protein content had higher IGF-1 than those receiving formula with a low protein content ${ }^{(31)}$. This is also thought to be one of the mechanisms behind the observed slightly slower growth of breast-fed compared with formula-fed infants ${ }^{(31)}$.

Potential mechanisms for the effects of FO on IGF-1 are speculative, but they may involve the sex hormones and the transcription factor PPAR- $\gamma$, which is activated by $n-3$ LCPUFA. Although testosterone and oestradiol levels are very low in children from about 6 months of age until onset of puberty ${ }^{(32)}$, these low levels, which may be undetectable by some assays, may still be important, as sex steroids accelerate growth during early childhood through stimulation of IGF-1 production, both directly and through effects on growth hormone secretion ${ }^{(33)}$. PPAR- $\gamma$ activation has been shown to inhibit the expression of aromatase ${ }^{(34)}$, the enzyme that converts testosterone to oestradiol. In females, most of the oestradiol is formed by the ovaries, whereas in males most of the oestradiol is produced by aromatisation of testosterone ${ }^{(35)}$. Reduced aromatase activity through the action of $n-3$ LCPUFA on PPAR- $\gamma$ may therefore affect IGF-1 production differently in boys and girls. However, these hypotheses are speculative and need further investigation.

The strengths of the present study include its randomised controlled design, the long intervention period and the use of erythrocyte EPA as a biomarker of $n-3$ LCPUFA intake, which showed good compliance with the relatively high dose of FO supplements. Although the study and sample size were not originally designed to look at sex interactions, and although the dose-response analyses showed modest correlations with $r$ values of approximately $0 \cdot 30$, the consistency between the results of the group comparisons and the dose-response analyses support their credibility. It would have been desirable to be able to investigate the potential effects on bone mass and body composition by use of DXA scans. However, this would have been costly and time-consuming, and the quality of the scans would have been questionable, as it requires the child to lie still without moving for at least $5 \mathrm{~min}$. In addition, measurement of a larger panel of growth and bone markers in blood and urine could have added more knowledge to the observed effects, but this was not obtainable with the available resources. The intake of $n$ - 3 LCPUFA is relatively high in
Denmark, and this may limit the potential impact of supplying additional n-3 LCPUFA. However, as demonstrated, the study population had a low intake of fish, and despite the high degree of breast-feeding, which is an important source of DHA in infancy ${ }^{(20)}$, their erythrocyte $n$-3 LCPUFA content was increased more than 2-fold by the FO supplementation. As risked in most FO trials in young children, blinding of parents was partly unsuccessful, probably because of the odours of FO when not kept at low temperature, and when spilled on the child's clothes. We tried to minimise this risk, as well as fatty acid oxidation, by instructing the parents to keep the unopened oil bottles in the freezer and in the refrigerator once opened. Although unblinding may be more of a problem in studies with adults or older children where the subjects themselves become aware of their allocation, it is a limitation of the present study that may have affected the parent's health behaviour and thereby potentially the results. Incorporating the oils into foods could have reduced this problem; however, this could have given other issues, as it is hard to administer a specific amount of the same food to an infant every day. Unfortunately, we did not ask the examiners to guess the children's group allocations. SO was chosen as control oil, as it most likely resembles the plant oil normally used in the infants' diets, and to avoid potential effects of an overall low supply of PUFA. EPA was used as a biomarker of FO intake and compliance, as it showed better correlation with estimated FO consumption than DHA. Erythrocytes were chosen because their $n$-3 LCPUFA content reflects intakes over the last months ${ }^{(36)}$ - that is, a relatively large part of the 9-month intervention period.

In conclusion, this study showed no effects of FO on the IGF axis in the total population of healthy Danish infants. However, plasma IGF-1 was higher in boys and IGFBP-3 was lower in girls after FO compared with SO supplementation from 9 to 18 months of age, and the results were supported by doseresponse relationships. However, although IGF-1 and IGFBP-3 were sex specifically and positively associated with length and BMI at 18 months, no sex-specific or overall effects of FO were seen on growth. These findings need confirmation in larger randomised trials, and the potential underlying mechanisms and long-term implications for growth and body composition should be investigated further.

\section{Acknowledgements}

We thank all the participating children and their families for their contribution to the study.

The EFiON study was supported by the Danish Council for Strategic Research, Programme Commission for Food and Health. The oil supplements were kindly provided by Axellus A/S, Lysaker, Norway.

A. D. A., K. F. M. and L. L. designed the study; A. D. A. and L. B. S. H. conducted the study; L. I. H. was responsible for the fatty acid analyses; C. T. D. and L. B. S. H. analysed the data; and C. T. D. and L. L. wrote the paper. C. T. D. had primary responsibility for the final content. All authors read and approved the final manuscript.

None of the authors reported conflicts of interest. 


\section{Supplementary material}

For supplementary material/s referred to in this article, please visit http://dx.doi.org/doi:10.1017/S0007114515004973

\section{References}

1. Mohan S \& Kesavan C (2012) Role of insulin-like growth factor-1 in the regulation of skeletal growth. Curr Osteoporos Rep 10, 178-186.

2. Hill DJ \& Hogg J (1989) Growth factors and the regulation of pre- and postnatal growth. Baillieres Clin Endocrinol Metab 3 579-625.

3. Juul A, Dalgaard P, Blum WF, et al. (1995) Serum levels of insulin-like growth factor (IGF)-binding protein-3 (IGFBP-3) in healthy infants, children, and adolescents: the relation to IGF-I, IGF-II, IGFBP-1, IGFBP-2, age, sex, body mass index, and pubertal maturation. J Clin Endocrinol Metab 80, $2534-2542$.

4. Juul A (2003) Serum levels of insulin-like growth factor I and its binding proteins in health and disease. Growth Horm IGF Res 13, 113-170.

5. Gatford KL, Egan AR, Clarke IJ, et al. (1998) Sexual dimorphism of the somatotrophic axis. J Endocrinol 157, 373-389.

6. Larnkjaer A, Molgaard C \& Michaelsen KF (2012) Early nutrition impact on the insulin-like growth factor axis and later health consequences. Curr Opin Clin Nutr Metab Care 15, 285-292.

7. Shen CL, Yeh JK, Rasty J, et al. (2006) Protective effect of dietary long-chain $n-3$ polyunsaturated fatty acids on bone loss in gonad-intact middle-aged male rats. Br J Nutr $\mathbf{9 5}$, 462-468.

8. Watkins BA, Li Y \& Seifert MF (2006) Dietary ratio of $n-6 / n-3$ PUFAs and docosahexaenoic acid: actions on bone mineral and serum biomarkers in ovariectomized rats. J Nutr Biochem 17, 282-289.

9. Griel AE, Kris-Etherton PM, Hilpert KF, et al. (2007) An increase in dietary $n-3$ fatty acids decreases a marker of bone resorption in humans. Nutr J $6,2$.

10. Andersen AD, Ludvig SE, Damsgaard CT, et al. (2013) The effect of fatty acid positioning in dietary triacylglycerols and intake of long-chain $n-3$ polyunsaturated fatty acids on bone mineral accretion in growing piglets. Prostaglandins Leukot Essent Fatty Acids 89, 235-240.

11. Coyne GS, Kenny DA \& Waters SM (2011) Effect of dietary $n-3$ polyunsaturated fatty acid supplementation on bovine uterine endometrial and hepatic gene expression of the insulin-like growth factor system. Theriogenology 75, 500-512.

12. Wei HK, Zhou Y, Jiang S, et al. (2013) Feeding a DHAenriched diet increases skeletal muscle protein synthesis in growing pigs: association with increased skeletal muscle insulin action and local mRNA expression of insulin-like growth factor 1. BrJ Nutr 110, 671-680.

13. Damsgaard CT, Molgaard C, Matthiessen J, et al. (2012) The effects of $n$-3 long-chain polyunsaturated fatty acids on bone formation and growth factors in adolescent boys. Pediatr Res 71, 713-719.

14. Asserhoj M, Nehammer S, Matthiessen J, et al. (2009) Maternal fish oil supplementation during lactation may adversely affect long-term blood pressure, energy intake, and physical activity of 7-year-old boys. J Nutr 139, 298-304.

15. Damsgaard CT, Eidner MB, Stark KD, et al. (2014) Eicosapentaenoic acid and docosahexaenoic acid in whole blood are differentially and sex-specifically associated with cardiometabolic risk markers in 8-11-year-old danish children. PLOS ONE 9, e109368.

16. Fong L, Muhlhausler BS, Gibson RA, et al. (2012) Perinatal maternal dietary supplementation of omega 3-fatty acids transiently affects bone marrow microenvironment, osteoblast and osteoclast formation, and bone mass in male offspring. Endocrinology 153, 2455-2465.

17. Pedersen L, Lauritzen L, Brasholt M, et al. (2012) Polyunsaturated fatty acid content of mother's milk is associated with childhood body composition. Pediatr Res $\mathbf{7 2}$, 631-636.

18. Andersen AD, Michaelsen KF, Hellgren LI, et al. (2011) A randomized controlled intervention with fish oil versus sunflower oil from 9 to 18 months of age: exploring changes in growth and skinfold thicknesses. Pediatr Res 70, 368-374.

19. Andersen AD, Molbak L, Michaelsen KF, et al. (2011) Molecular fingerprints of the human fecal microbiota from 9 to 18 months old and the effect of fish oil supplementation. J Pediatr Gastroenterol Nutr 53, 303-309.

20. Harslof LB, Larsen LH, Ritz C, et al. (2013) FADS genotype and diet are important determinants of DHA status: a crosssectional study in Danish infants. Am J Clin Nutr 97, $1403-1410$

21. Harslof LB, Damsgaard CT, Hellgren LI, et al. (2014) Effects on metabolic markers are modified by PPARG2 and COX2 polymorphisms in infants randomized to fish oil. Genes Nutr 9, 396.

22. Harslof LB, Damsgaard CT, Andersen AD, et al. (2014) Reduced ex vivo stimulated IL- 6 response in infants randomized to fish oil from 9 to 18 months, especially among PPARG2 and COX2 wild types. Prostaglandins Leukot Essent Fatty Acids 94, 21-27.

23. Gondolf UH, Tetens I, Hills AP, et al. (2012) Validation of a pre-coded food record for infants and young children. Eur J Clin Nutr 66, 91-96.

24. Lauritzen L, Jorgensen MH, Mikkelsen TB, et al. (2004) Maternal fish oil supplementation in lactation: effect on visual acuity and $n-3$ fatty acid content of infant erythrocytes. Lipids 39, 195-206.

25. Larnkjaer A, Hoppe C, Molgaard C, et al. (2009) The effects of whole milk and infant formula on growth and IGF-I in late infancy. Eur J Clin Nutr 63, 956-963.

26. Gholamhosseini S, Nematipour E, Djazayery A, et al. (2015) Omega-3 fatty acid differentially modulated serum levels of IGF1 and IGFBP3 in men with CVD: a randomized, double-blind placebo-controlled study. Nutrition 31, 480-484

27. Collins CT, Makrides M, Gibson RA, et al. (2011) Pre- and post-term growth in pre-term infants supplemented with higher-dose DHA: a randomised controlled trial. Br J Nutr 105, 1635-1643.

28. Makrides M, Gibson RA, Udell T, et al. (2005) Supplementation of infant formula with long-chain polyunsaturated fatty acids does not influence the growth of term infants. Am J Clin Nutr 81, 1094-1101.

29. Closa-Monasterolo R, Ferre N, Luque V, et al. (2011) Sex differences in the endocrine system in response to protein intake early in life. Am J Clin Nutr 94, 1920S-1927S.

30. Makrides M, Gibson RA, McPhee AJ, et al. (2010) Effect of DHA supplementation during pregnancy on maternal depression and neurodevelopment of young children: a randomized controlled trial. JAMA 304, 1675-1683.

31. Socha P, Grote V, Gruszfeld D, et al. (2011) Milk protein intake, the metabolic-endocrine response, and growth in infancy: data from a randomized clinical trial. Am J Clin Nutr 94, 1776S-1784S. 
32. Andersson AM, Toppari J, Haavisto AM, et al. (1998) Longitudinal reproductive hormone profiles in infants: peak of inhibin B levels in infant boys exceeds levels in adult men. $J$ Clin Endocrinol Metab 83, 675-681.

33. Juul A (2001) The effects of oestrogens on linear bone growth. Hum Reprod Update 7, 303-313.

34. Margalit O, Wang D \& Dubois RN (2012) PPARgamma agonists target aromatase via both PGE2 and BRCA1. Cancer Prev Res (Phila) 5, 1169-1172.
35. Roche AF \& Shumei SS (2003) Determinants of growth. In Human Growth-Assessment and Interpretation, pp. 111-171 [AF Roche and SS Shumei, editors]. Cambridge: Cambridge University Press.

36. Katan MB, Deslypere JP, van Birgelen AP, et al. (1997) Kinetics of the incorporation of dietary fatty acids into serum cholesteryl esters, erythrocyte membranes, and adipose tissue: an 18-month controlled study. J Lipid Res $\mathbf{3 8}$, 2012-2022. 